computers do very well against any but well- versed IM strength players.

Well, the tournament in The Hague was won by two human players who are not at all of IM strength. When analyzing the games, not just gloating over the results, it becomes evident that the reasons for their victory are that these human beings took their opponents seriously and refrained from experimentation.

I think you are right in saying that computers are still incapable of playing a well-conceived strategic game. And this is also the reason why I still believe, as I stated four years ago, that I have little to fear from computers, even though I don't play much anymore. It will take ages, if ever, before a computer is capable of beating a good human chess-player under tournament conditions. (In blitz chess and rapid chess computers may win some games.)

\title{
MUCH TO FEAR FROM CHESS COMPUTERS
}

\author{
Prof. dr. H. Berliner \\ Computer Science Department \\ Carnegie-Mellon University \\ Pittsburgh, PA 15213 \\ USA
}

"After reading Mr. Bakker's note several times, I am at a loss to understand what it is about. My only explanation is that either:

1) he is upset at the last paragraph of my article, or

2) he really does not understand too much about chess.

I will reject the latter as an explanation since he seems to be a master level player. Now let us come down to the crux of the matter. Mr. Bakker made a statement in the open literature (ICCA Journal, 1985 [Vol. 8, No. 4, p. 260]) that "he did not expect to have anything to fear from computers for the next 5 years". That was based on a single encounter with Cray Blitz, a program that all connoisseurs know has done miserably against human competition in the Fredkin matches. Therefore, I found it insulting to have the whole field judged by the performance of Cray Blitz, especially at a time when Hitech had just won the ACM tournament with a perfect score.

Now one could take issue with what "nothing to fear" means, but I believe all will interpret the statement to mean that he does not expect to lose to computers at least until 1990. However, I challenged him to turn his expression of confidence into a bet, and he declined this (ICCA Journal, Vol. 9, No. 2, p. 111 and 125). It seems rather clear to me that he or his backers would lose whatever money they wagered, very likely against Hitech, and certainly to Deep Thought.

So why is Mr. Bakker changing the argument to "computers beating good players" all of a sudden. "Good players" is as non-quantitive as "nothing to fear". Mr. Bakker seems to revel in making statements that are imprecise, and when one tries to challenge him to make them precise, he quickly runs away.

That is all I have to say on the matter. If Mr. Bakker wishes to make ridiculous statements in the press, then he must be willing to "put his money where his mouth is". Failing to do that, he appears to be falling back on writing meaningless letters to the editor."

\section{HELP WANTED: KRP(a)KB}

\author{
Lars Falk \\ Tegnérgatan $34 \mathrm{~B}$ \\ 75227 Uppsala \\ Sweden
}

From Dr. Lars Falk, we received the following suggestion for exploiting a database innovatively. The endgame considered is $\mathrm{KRP}(\mathrm{a}) \mathrm{KB}$. Quite recently dr. Falk discovered that a Russian composer, Rezvov, has found a complicated win in this endgame based on a series of Zugzwang positions. To quote him verbatim: 
"This has the interesting consequence that most study ideas are ruled out, because you can always find additional solutions based on the method indicated by Rezvov. It would also be interesting to know whether his method is unique; otherwise even Rezvov's study would be incorrect in the sense of study composition!

To clarify my meaning consider the position $\mathrm{Kc} 2, \mathrm{Re} 2, \mathrm{~Pa} 6$ - Kb6, Bal. According to theory Black can draw this position easily if he can reach the diagonal g1-a7 with his Bishop, but in the present case White has a studylike win by $1 . \mathrm{Kd} 3$ ! (White covers $\mathrm{d} 4$ and threatens Ra2; the Bishop has only one field) Bh8 2. Re8! Ba1 (once more the only field; after forcing Black to pass the diagonal twice White can now win by Zugzwang) 3. Kc4! Ka7 (now the Bishop has no moves at all) 4. Rd8! (threatens Kb5) Bf6 5. Rd7+! Ka8 6. a7 followed by Kc4-b5-a6 winning.

All this is impressive and possibly near optimal but even if White plays inefficiently he can probably win, though in a very complicated manner. Say that White plays sloppily, for instance 1 . Re6+ Ka7 2. Kd3 Bh8 3 . $\mathrm{Kc} 4 \mathrm{Bb} 2$. According to Rezvov's analysis he can still win by $4 . \mathrm{Kd} 5$ ! looking for mating threats at the right moment. The main line according to Rezvov is 4. Kd5! Bc3 5. Re3! Bb2 6. Rb3! Bf6! 7. Rh4! Bb2 8. Rh6 Bc3 9. Re6!! (Zugzwang; this is the same position as at move 4 but with Black to move) 9. ... Bb2 10. Rc6!! (Zugzwang, forcing the Bishop into the corner) 10. ... Bh8 11. Rd6! Bc3 12. Kc6 Kxa6 13. Rd3 Bb4 14. Rd1 winning.

I have two doubts. The first one is about the analysis itself: is it correct? The second one: what are configurations which would satisfy a problemist's wishes for the position to be free from duals? Probably only the computer can tell. The Russian endgame theoretician Kopnin has been analysing this material for some years and will probably publish an analysis of the a-Pawn, too. So far he has only written about b-, c-and d-Pawns. I think it would be an excellent idea to cover the same field with a computer, since this endgame is exactly of the type where the database excels, i.e., where there is a large number of Zugzwang positions."

\title{
A WIN BY KARPOV JUSTIFIED
}

\author{
Lars Rasmussen \\ Jegstrupvaenget 409 \\ 8310 Tranbjerg \\ Denmark
}

We have received the following note by Rasmussen, pointing out a potential correction to a statement of Vol. 12, No. 3, p. 116. The Correspondent disputes that Karpov should have won from a drawn position and contends that the win was computable. We quote:

"The game referred to must be Karpov - Ftačník, in which the following position arose with Black to move: Ke6, Rf7 - Kh3, Nb6. White's last move was to capture a Pawn on e6. Play continued 83. ... Nc4 84. Rf3 Kg4 85. Rd3! Kg5 86. Kd5 Nb6 87. Ke5 Nc4 88. Ke4 Nb6 89. Rd8 Nc4 90. Rd4 Nb6 91. Ke5 Nc8 92. Ke6 Na7 93. $\mathrm{Kd} 7$ and Black resigned.

In his notes in Chess Informant (Vol. 46, game 38) Karpov suggests that 83. ... Kg3 84. Rc7 Kf3 should lead to a draw. However, White wins with 85. Rc3 Ke2 (85. ... Ke4 86. Rc6 Nd5 87. Rc4) 86. Rc6 Na4 87. Rc4 Nb6 88. Rb4 Nc8 89. Kd7. In fact, the initial KRKN position is not a draw, but a win for White. 